\title{
An Algorithm for Road Coloring
}

\author{
A.N. Trahtman* \\ Bar-Ilan University, Dep. of Math., 52900, Ramat Gan, Israel \\ Lect. Notes in Comput. Sci., 7056, 349-360. Springer 2011, IWOCA 2011
}

\begin{abstract}
A coloring of edges of a finite directed graph turns the graph into a finite-state automaton. The synchronizing word of a deterministic automaton is a word in the alphabet of colors (considered as letters) of its edges that maps the automaton to a single state. A coloring of edges of a directed graph of uniform outdegree (constant outdegree of any vertex) is synchronizing if the coloring turns the graph into a deterministic finite automaton possessing a synchronizing word.

The road coloring problem is the problem of synchronizing coloring of a directed finite strongly connected graph of uniform outdegree if the greatest common divisor of the lengths of all its cycles is one. The problem posed in 1970 has evoked noticeable interest among the specialists in the theory of graphs, automata, codes, symbolic dynamics as well as among the wide mathematical community.

A polynomial time algorithm of $O\left(n^{3}\right)$ complexity in the worst case and quadratic in the majority of studied cases for the road coloring of the considered graph is presented below. The work is based on the recent positive solution of the road coloring problem. The algorithm was implemented in the freeware package TESTAS.
\end{abstract}

Keywords: algorithm, road coloring, graph, deterministic finite automaton, synchronization

\section{Introduction}

The road coloring problem was stated almost 40 years ago [2], [1] for a strongly connected directed finite deterministic graph of uniform outdegree where the greatest common divisor (gcd) of the lengths of all its cycles is one. The edges of the graph being unlabelled, the task is to find a labelling of the edges that turns the graph into a deterministic finite automaton possessing a synchronizing word. The outdegree of the vertex can be considered also as the size of an alphabet where the letters denote colors.

The condition on gcd is necessary [1], [9]. It can be replaced by the equivalent property that there does not exist a partition of the set of vertices on subsets $V_{1}, V_{2}, \ldots, V_{k}=V_{1}(k>2)$ such that every edge which begins in $V_{i}$ has its end in $V_{i+1}$ [9], [16].

\footnotetext{
^ Email: trakht@macs.biu.ac.il
} 
Together with the Černy conjecture [7], [8], [15], [20] the road coloring problem used to belong to the most fascinating problems in the theory of finite automata. The popular Internet Encyclopedia "Wikipedia" mentioned it many years on the list of the most interesting unsolved problems in mathematics.

For some results in this area, see [5], [6], [10], [11], [12], [13], [14], [16], [17], [19]. A detailed history of investigations can be found in [6]. The final positive solution of the problem is stated in [24].

An algorithm for road coloring oriented on DNA computing [13] is based on the massive parallel computing of sequences of length $O\left(n^{3}\right)$. The implementation of the algorithm as well as the implementation of effective DNA computing is still an open problem.

Another new algorithm for road coloring (ArXiv [4]) as well as our algorithm below is based on the proof of [24]. This proof is constructive and leads to an algorithm that finds a synchronized labelling with cubic worst-case time complexity. Both of the above mentioned algorithms use concepts and ideas of the considered proof together with the concepts from [9], [14], but use different methods to reduce the time complexity. A skillful study of the graph was added in [4].

The presented algorithm for the road coloring (see also ArXiv [22]) reduces the time complexity with the help of the study of two cycles with common vertex (Lemma 10). It gives us the possibility to reduce quite often the time complexity.

The theorems and lemmas from [24] and [23] are presented below without proof. The proofs are given only for new (or modified) results. The time complexity of the algorithm for a graph with $n$ vertices and $d$ outgoing edges of any vertex is $O\left(n^{3} d\right)$ in the worst case and quadratic in the majority of the studied cases. The space complexity is quadratic. This is the first embedded algorithm for road coloring.

The description of the algorithm is presented below together with some pseudo codes of the implemented subroutines. The algorithm is implemented in the freeware package TESTAS (http://www.cs.biu.ac.il/ trakht/syn.html) [25]. The easy access to the package ensures the possibility to everybody to verify the considered algorithm.

The role of the road coloring is substantial also in education. "The Road Coloring Conjecture makes a nice supplement to any discrete mathematics course" [18]. The realization of the algorithm is demonstrated on the basis of a linear visualization program [25] and can analyze any kind of input graph.

\section{Preliminaries}

As usual, we regard a directed graph with letters assigned to its edges as a finite automaton, whose input alphabet $\Sigma$ consists of these letters. The graph is called a transition graph of the automaton. The letters from $\Sigma$ can be considered as colors and the assigning of colors to edges will be called coloring. 
A finite directed strongly connected graph with constant outdegree of all its vertices where the gcd of lengths of all its cycles is one will be called an $A G W$ graph (as introduced by Adler, Goodwyn and Weiss).

We denote by $|P|$ the size of the subset $P$ of states of an automaton (of vertices of a graph).

If there exists a path in an automaton from the state $\mathbf{p}$ to the state $\mathbf{q}$ and the edges of the path are consecutively labelled by $\sigma_{1}, \ldots, \sigma_{k}$, then for $s=\sigma_{1} \ldots \sigma_{k} \in$ $\Sigma^{+}$we shall write $\mathbf{q}=\mathbf{p} s$.

Let $P s$ be the set of states $\mathbf{p} s$ for $\mathbf{p} \in P, s \in \Sigma^{+}$. For the transition graph $\Gamma$ of an automaton, let $\Gamma s$ denote the map of the set of states of the automaton.

A word $s \in \Sigma^{+}$is called a synchronizing word of the automaton with transition graph $\Gamma$ if $|\Gamma s|=1$.

A coloring of a directed finite graph is synchronizing if the coloring turns the graph into a deterministic finite automaton possessing a synchronizing word.

Bold letters will denote the vertices of a graph and the states of an automaton.

A pair of distinct states $\mathbf{p}, \mathbf{q}$ of an automaton (of vertices of the transition graph) will be called synchronizing if $\mathbf{p} s=\mathbf{q} s$ for some $s \in \Sigma^{+}$. In the opposite case, if $\mathbf{p} s \neq \mathbf{q} s$ for any $s$, we call the pair a deadlock.

A synchronizing pair of states $\mathbf{p}, \mathbf{q}$ of an automaton is called stable if for any word $u$ the pair $\mathbf{p} u, \mathbf{q} u$ is also synchronizing [9], [14].

We call the set of all outgoing edges of a vertex a bunch if all these edges are incoming edges of only one vertex.

The subset of states (of vertices of the transition graph $\Gamma$ ) of maximal size such that every pair of states from the set is a deadlock will be called an F-clique.

\section{Some properties of $F$-cliques and stable pairs}

The road coloring problem was formulated for $A G W$ graphs [1] and only such graphs are considered in Sections 1 and 2.

Let us recall that a binary relation $\rho$ on the set of the states of an automaton is called congruence if $\rho$ is equivalence and for any word $u$ from $\mathbf{p} \rho \mathbf{q}$ follows $\mathbf{p} u$ $\rho \mathbf{q} u$. Let us formulate an important result from [9], [14] in the following form:

Theorem 1 [14] Let us consider a coloring of an $A G W$ graph $\Gamma$. Let $\rho$ be the transitive and reflexive closure of the stability relation on the obtained automaton. Then $\rho$ is a congruence relation, $\Gamma / \rho$ is also an $A G W$ graph and a synchronizing coloring of $\Gamma / \rho$ implies a synchronizing recoloring of $\Gamma$.

Lemma 1 [24], [9] Let $F$ be an F-clique of some coloring of an AGW graph $\Gamma$. For any word s the set $F s$ is also an F-clique and any state $\mathbf{p}$ belongs to some F-clique.

Lemma 2 Let $A$ and $B$ (with $|A|>1$ ) be distinct $F$-cliques of some coloring of an $A G W$ graph $\Gamma$ such that $|A|-|A \cap B|=1$. Then for all $\mathbf{p} \in A \backslash A \cap B$ and $\mathbf{q} \in B \backslash A \cap B$, the pair $(\mathbf{p}, \mathbf{q})$ is stable. 
Proof. By the definition of an $F$-clique, $|A|=|B|$ and $|B|-|A \cap B|=1$, too. If the pair of states $\mathbf{p} \in A \backslash B$ and $\mathbf{q} \in B \backslash A$ is not stable, then for some word $s$ the pair $(\mathbf{p} s, \mathbf{q} s)$ is a deadlock. Any pair of states from the $F$-clique $A$ and from the $F$-clique $B$, as well as from the $F$-cliques $A s$ and $B s$, is a deadlock. So any pair of states from the set $(A \cup B) s$ is a deadlock. One has $|(A \cup B) s|=|A s|+1=|A|+1>|A|$. So the size of the set $(A \cup B) s$ of deadlocks is greater than the maximal size of $F$-clique. Contradiction.

Lemma 3 If some vertex of an $A G W$ graph $\Gamma$ has two incoming bunches, then the origins of the bunches form a stable pair by any coloring.

Proof. If a vertex $\mathbf{p}$ has two incoming bunches from $\mathbf{q}$ and $\mathbf{r}$, then the couple $\mathbf{q}$, $\mathbf{r}$ is stable for any coloring because $\mathbf{q} \sigma=\mathbf{r} \sigma=\mathbf{p}$ for any $\sigma \in \Sigma$.

\section{The spanning subgraph of an $A G W$ graph}

Definition 4 Let us call a subgraph $S$ of an $A G W$ graph $\Gamma$, a spanning subgraph of $\Gamma$, if $S$ contains all vertices of $\Gamma$ and if each vertex has exactly one outgoing edge. (In usual graph-theoretic terms it is a 1-outregular spanning subgraph).

A maximal subtree of a spanning subgraph $S$ with its root on a cycle from $S$ and having no common edges with the cycles of $S$ is called a tree of $S$.

The length of a path from a vertex $\mathbf{p}$ through the edges of the tree of the spanning set $S$ to the root of the tree is called a level of $\mathbf{p}$ in $S$.

A tree with a vertex of maximal level is called a maximal tree.

Remark 5 Any spanning subgraph $S$ consists of disjoint cycles and trees with roots on the cycles. Any tree and cycle of $S$ is defined identically. The level of the vertices belonging to some cycle is zero. The vertices of the trees except the roots have positive level. The vertices of maximal positive level have no incoming edge in $S$. The edges labelled by a given color defined by any coloring form a spanning subgraph. Conversely, for each spanning subgraph, there exists a coloring and a color such that the set of edges labelled with this color corresponds to this spanning subgraph.

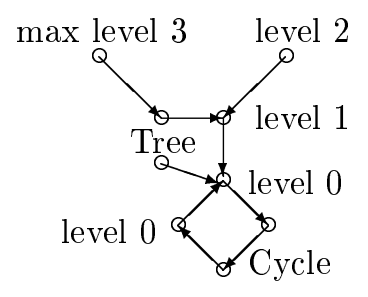

Lemma 6 [24] Let $N$ be a set of vertices of maximal level in some tree of the spanning subgraph $S$ of an $A G W$ graph $\Gamma$. Then, via a coloring of $\Gamma$ such that all edges of $S$ have the same color $\alpha$, for any $F$-clique $F$ holds $|F \cap N| \leq 1$. 
Lemma 7 [24] Let $\Gamma$ be an $A G W$ graph with a spanning subgraph $R$ which is a union of cycles (without trees). Then the non-trivial graph $\Gamma$ has another spanning subgraph with exactly one maximal tree.

Lemma 8 Let $R$ be a spanning subgraph of an $A G W$ graph $\Gamma$. Let $T$ be a maximal tree of $R$ with a vertex $\mathbf{p}$ of maximal positive level $L$ and with a root $\mathbf{r}$ on a cycle $H$ of $R$. Let us change the spanning subgraph by means of the following flips:

1)an edge $\bar{a}=\mathbf{a} \rightarrow \mathbf{p}$ replaces the edge $\bar{d}=\mathbf{a} \rightarrow \mathbf{d}$ of $R$ for appropriate vertices $\mathbf{a}$ and $\mathbf{d} \neq \mathbf{p}$,

2) replacing edge $\bar{b}=\mathbf{b} \rightarrow \mathbf{r}$ of $T$ by an edge $\mathbf{b} \rightarrow \mathbf{x}$ for appropriate vertices $\mathbf{b}$ and $\mathbf{x} \neq \mathbf{r}$,

3) replacing edge $\bar{c}=\mathbf{c} \rightarrow \mathbf{r}$ of $H$ by an edge $\mathbf{c} \rightarrow \mathbf{x}$ for appropriate vertices c and $\mathbf{x} \neq \mathbf{r}$.

Suppose that one or two consecutive flips do not increase the number of edges in cycles (Condition ${ }^{*}$ ) and no vertex of $\Gamma$ has two incoming bunches (Condition**). Then there exists a spanning subgraph with a single maximal non-trivial tree.

Proof. In view of Lemma 7, suppose that $R$ has non-trivial trees. Further consideration is necessary only if the maximal tree $T$ is not single.

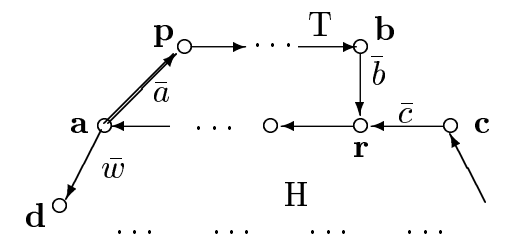

Our aim is to increase the maximal level $L$ using the three aforesaid flips. If one of the flips does not succeed, let us go to the next, assuming the situation in which the previous fails, and excluding the successfully studied cases. We check at most two flips together. Let us begin from

the edge $\bar{a}$ ) Suppose first $\mathbf{a} \notin H$. If $\mathbf{a}$ belongs to the path in $T$ from $\mathbf{p}$ to $\mathbf{r}$ then a new cycle with part of the path and the edge $\mathbf{a} \rightarrow \mathbf{p}$ is added to $R$ extending the number of vertices in its cycles in spite of Condition* of lemma. In the opposite case the level of $\mathbf{a}$ is $L+1$ in a single maximal tree.

So let us assume $\mathbf{a} \in H$. In this case the vertices $\mathbf{p}, \mathbf{r}$ and $\mathbf{a}$ belong to a cycle $H_{1}$ of a new spanning subgraph $R_{1}$ obtained by removing $\bar{d}$ and adding $\bar{a}$. So we have the cycle $H_{1} \in R_{1}$ instead of $H \in R$. If the length of the path from $\mathbf{r}$ to $\mathbf{a}$ in $H$ is $r_{1}$, then $H_{1}$ has length $L+r_{1}+1$. A path from $\mathbf{r}$ to $\mathbf{d}$ of the cycle $H$ remains in $R_{1}$. Suppose that its length is $r_{2}$. So the length of the cycle $H$ is $r_{1}+r_{2}+1$. The length of the cycle $H_{1}$ is not greater than the length of $H$ in view of Condition*. So $r_{1}+r_{2}+1 \geq L+r_{1}+1$, whence $r_{2} \geq L$. If $r_{2}>L$, then the length $r_{2}$ of the path from $\mathbf{d}$ to $\mathbf{r}$ in a tree of $R_{1}$ (as well as the level of $\mathbf{d}$ ) is greater than $L$. The tree containing $\mathbf{d}$ is the desired single maximal tree.

So we can assume for further consideration that $L=r_{2}$ and $\mathbf{a} \in H$. An analogous statement can be stated for any maximal tree. 
the edge $\bar{b}$ ) Suppose that the set of outgoing edges of the vertex $\mathbf{b}$ is not $\mathbf{a}$ bunch. So one can replace in $R$ the edge $\bar{b}$ by an edge $\bar{v}=\mathbf{b} \rightarrow \mathbf{v}(\mathbf{v} \neq \mathbf{r})$.

The vertex $\mathbf{v}$ could not belong to $T$ because in this case a new cycle is added to $R$ in spite of Condition*.

If the vertex $\mathbf{v}$ belongs to another tree of $R$ but not to the cycle $H$, then $T$ is a part of a new tree $T_{1}$ with a new root of a new spanning subgraph $R_{1}$ and the path from $\mathbf{p}$ to the new root has a length greater than $L$. Therefore the tree $T_{1}$ is the unique maximal tree in $R_{1}$.

If $\mathbf{v}$ belongs to some cycle $H_{2} \neq H$ in $R$, then together with replacing $\bar{b}$ by $\bar{v}$, we also replace the edge $\bar{d}$ by $\bar{a}$. So we extend the path from $\mathbf{p}$ to the new root $\mathbf{v}$ of $H_{2}$ at least by the edge $\bar{a}=\mathbf{a} \rightarrow \mathbf{p}$ and there is a unique maximal tree of level $L_{1}>L$ which contains the vertex $\mathbf{d}$.

Now it remains only the case when $\mathbf{v}$ belongs to the cycle $H$. The vertex $\mathbf{p}$ also has level $L$ in a new tree $T_{1}$ with root $\mathbf{v}$. The only difference between $T$ and $T_{1}$ (just as between $R$ and $R_{1}$ ) is the root and the incoming edge of this root. The new spanning subgraph $R_{1}$ has the same number of vertices in their cycles just as does $R$. Let $r_{2}^{\prime}$ be the length of the path from $\mathbf{d}$ to $\mathbf{v} \in H$.

For the spanning subgraph $R_{1}$, one can obtain $L=r_{2}^{\prime}$ just as it was done earlier in the case of the edge $\bar{a}$ ) for $R$. From $\mathbf{v} \neq \mathbf{r}$ follows $r_{2}^{\prime} \neq r_{2}$, though $L=r_{2}^{\prime}$ and $L=r_{2}$.

So for further consideration suppose that the set of outgoing edges of the vertex $\mathbf{b}$ is a bunch to $\mathbf{r}$.

The edge $\bar{c}$ ) The set of outgoing edges of the vertex $\mathbf{c}$ is not a bunch in virtue of Condition** ( $\mathbf{r}$ has another bunch from $\mathbf{b}$.)

Let us replace in $R$ the edge $\bar{c}$ by an edge $\bar{u}=\mathbf{c} \rightarrow \mathbf{u}$ such that $\mathbf{u} \neq \mathbf{r}$. The vertex $\mathbf{u}$ could not belong to the tree $T$ because one has in this case a cycle with all vertices from $H$ and some vertices of $T$ whence its length is greater than $|H|$ and so the number of vertices in the cycles of a new spanning subgraph grows in spite of Condition*.

If the vertex $\mathbf{u}$ does not belong to $T$, then the tree $T$ is a part of a new tree with a new root. The path from $\mathbf{p}$ to the new root is extended at least by a part of $H$ starting at the former root $\mathbf{r}$. The new level of $\mathbf{p}$ therefore is maximal and greater than the level of any vertex in another tree.

Thus in any case we obtain a spanning subgraph with a single non-trivial maximal tree.

Lemma 9 For some coloring of any $A G W$ graph $\Gamma$, there exists a stable pair of states.

Proof. We exclude the case of two incoming bunches of a vertex in virtue of Lemma 3. There exists a coloring such that for some color $\alpha$, the corresponding spanning subgraph $R$ has maximum edges in cycles.

By Lemma 8, we must consider now a spanning subgraph $R$ with a single maximal tree $T$. Let the root $\mathbf{r}$ of $T$ belong to the cycle $C$.

By Lemma 1, in a strongly connected transition graph for every word $s$ and $F$-clique $F$ of size $|F|>1$, the set $F s$ also is an $F$-clique of the same size and for any state $\mathbf{p}$ there exists an $F$-clique $F$ such that $\mathbf{p} \in F$. 
In particular, some $F$-clique $F$ has a non-empty intersection with the set $N$ of vertices of maximal level $L$. The set $N$ belongs to one tree, whence by Lemma $6|N \cap F|=1$. Let $\mathbf{p} \in N \cap F$.

The word $\alpha^{L-1}$ maps $F$ on an $F$-clique $F_{1}$ of size $|F|$. One has $\left|F_{1} \backslash C\right|=1$ because any sequence of length $L-1$ of edges of color $\alpha$ in any tree of $R$ leads to a cycle. For the set $N$ of vertices of maximal level, $N \alpha^{L-1} \nsubseteq C$ holds. So $\left|N \alpha^{L-1} \cap F_{1}\right|=\left|F_{1} \backslash C\right|=1, \mathbf{p} \alpha^{L-1} \in F_{1} \backslash C$ and $\left|C \cap F_{1}\right|=\left|F_{1}\right|-1$.

Let the integer $m$ be a common multiple of the lengths of all considered cycles colored by $\alpha$. So for any $\mathbf{r}$ in $C$ as well as in $F_{1} \cap C$ holds $\mathbf{r} \alpha^{m}=\mathbf{r}$. Let $F_{2}$ be $F_{1} \alpha^{m}$. We have $F_{2} \subseteq C$ and $C \cap F_{1}=F_{1} \cap F_{2}$.

Thus the two $F$-cliques $F_{1}$ and $F_{2}$ of size $\left|F_{1}\right|>1$ have $\left|F_{1}\right|-1$ common vertices. So $\left|F_{1} \backslash\left(F_{1} \cap F_{2}\right)\right|=1$, whence by Lemma 2 , the pair of states $\mathbf{p} \alpha^{\mathbf{L}-1}$ from $F_{1} \backslash\left(F_{1} \cap F_{2}\right)$ and $\mathbf{q}$ from $F_{2} \backslash\left(F_{1} \cap F_{2}\right)$ is stable. It is obvious that $\mathbf{q}=\mathbf{p} \alpha^{\mathbf{L}+\mathbf{m - 1}}$.

\section{Theorem 2 [24] Every AGW graph has a synchronizing coloring.}

Theorem 3 [23] Let every vertex of a strongly connected directed graph $\Gamma$ have the same number of outgoing edges. Then $\Gamma$ has synchronizing coloring if and only if the greatest common divisor of lengths of all its cycles is one.

The goal of the following lemma is to reduce the complexity of the algorithm.

Lemma 10 Let $\Gamma$ be an $A G W$ graph having two cycles $C_{u}$ and $C_{v}$. Suppose that either $C_{u} \cap C_{v}=\left\{\mathbf{p}_{1}\right\}$ or $C_{u} \cap C_{v}=\left\{\mathbf{p}_{k}, \ldots, \mathbf{p}_{1}\right\}$, where all incoming edges of $\mathbf{p}_{i}$ develop a bunch from $\mathbf{p}_{i+1}(i<k)$.

Let $u \in C_{u}$ and $v \in C_{v}$ be the distinct edges of the cycles $C_{u}$ and $C_{v}$ leaving $\mathbf{p}_{1}$. Let $R_{u}$ be a spanning subgraph with all edges from $C_{u}$ and $C_{v}$ except $u$. The spanning subgraph $R_{v}$ is obtained from $R_{u}$ by removing $v$ and adding $u$.

Then at least one of two spanning subgraphs $R_{u}, R_{v}$ has a unique maximal tree whose root is $\mathbf{p}_{1}$.

Proof. Let us add to $R_{u}$ the edge $u$ and consider a set of trees with roots on the cycles $C_{u}$ and $C_{v}$. The trees have no common vertices and have no vertices except a root on the cycles $C_{u}$ and $C_{v}$. The same set of trees can be obtained by adding the edge $v$ to $R_{v}$.

Let us define the levels of vertices of a tree as in the case of a spanning subgraph and consider the set of maximal trees (the trees with a maximal vertex level).

If all maximal trees have a common root, then $R_{u}$ (and also $R_{v}$ ) is a spanning subgraph with a unique maximal tree.

If maximal trees have different roots, then let as take a maximal tree $T$ with root $\mathbf{r}$ such that the length of the path $P$ from $\mathbf{r}$ to $\mathbf{p}_{1}$ on the cycle $C_{u}$ (or $C_{v}$ ) is maximal. If $P$ belongs to $C_{u}$, then the tree $T$ is extended by the path $P$, whence $R_{u}$ has a unique maximal tree. In the opposite case, $R_{v}$ has a unique maximal tree. 


\section{The algorithm for synchronizing coloring}

Let us start with transition graph of an arbitrary deterministic complete finite automaton.

\subsection{Preliminary steps}

The study is based on Theorem 3. A synchronizing graph has a sink strongly connected component $(S C C)$. Our aim is to reduce the study to sink $S C C$ (if it exists) in order to remove non-synchronizing graphs without sink $S C C$ and then check the condition on $\mathrm{gcd}$.

The function CheckSinkSCC verifies the existence of sink $S C C$. We use the linear algorithm for finding strongly connected components $S C C$ [3], [21].

Then we remove all $S C C$ as having outgoing edges to other $S C C$. If only one $S C C$ remains then let us continue. In the opposite case a synchronizing coloring does not exist.

We study a strongly connected graph (with one $S C C$ ). The function FindGCDofCycles finds the great common divisor $(\mathrm{gcd})$ of lengths of cycles of the automaton and verifies the necessary conditions of synchronizability $(g c d=1)$.

Let $\mathbf{p}$ be an arbitrary fixed vertex. Suppose $d(\mathbf{p})=1$. Then we use a depthfirst search from $\mathbf{p}$. For an edge $\mathbf{r} \rightarrow \mathbf{q}$ where $d(\mathbf{r})$ is already defined and $d(\mathbf{q})$ is not, suppose $d(\mathbf{q})=d(\mathbf{r})+1$. If $d(\mathbf{q})$ is defined, let us add the non-zero difference $a b s(d(\mathbf{q})-1-d(\mathbf{r}))$ to the set $D$. The integer from $D$ is a difference of lengths of two paths from $\mathbf{p}$ to $\mathbf{q}$. In a strongly connected graph, the $g c d$ of all elements of $D$ is also a $g c d$ of lengths of all cycles [2], [23].

If $g c d=1$ for all integers from $D$, then the graph has synchronizing coloring. In opposite case the answer is negative. So we reduce the investigation to an $A G W$ graph.

Let us proceed with an arbitrary coloring of such a graph $\Gamma$ with $n$ vertices and constant outdegree $d$. The considered $d$ colors define $d$ spanning subgraphs of the graph.

We keep the preimages of vertices and colored edges by any transformation and homomorphism.

If there exists a loop in $\Gamma$ around a state $\mathbf{r}$, then let us color the edges of a tree whose root is $\mathbf{r}$ with the same color as the color of the loop. The other edges may be colored arbitrarily. The coloring is synchronizing [1]. The function FindLoopColoring finds the coloring.

\subsection{Help subroutines}

In the case of two incoming bunches of some vertex, the origins of these bunches develop a stable pair by any coloring (Lemma 3). We merge both vertices in the homomorphic image of the graph (Theorem 1) and obtain according to the theorem a new $A G W$ graph of a smaller size. The pseudo code of corresponding procedure returns two such origins of bunches (a stable pair). 
The linear search of two incoming bunches and of the loop can be made at any stage of the algorithm.

The function HomonorphicImage of linear complexity reduces the size of the considered automaton and its transition graph. The congruence classes of the homomorphism are defined by a stable pair (Theorem 1). A new $A G W$ graph of a smaller size will be the output.

The main part of the algorithm needs the parameters of the spanning subgraph: levels of all vertices, the number of vertices (edges) in cycles, trees, next and former vertices. We keep the tree and the cycle of any vertex, the root of the tree. We form the set of vertices of maximal level and the set of maximal trees. The function FindParameters (spanning subgraph $S$, parameters) is linear and used by any recoloring step.

The subroutine MaximalTreeToStablePair of linear complexity finds a stable pair in a given spanning subgraph with unique maximal tree. The stable pair consists of two beginnings of incoming edges of the root of the unique maximal tree (Lemma 9).

\subsection{A possibility to reduce the complexity}

Our algorithm as well as the algorithm of [4] is based on [24]. Only this section essentially differs in both these papers.

If there are two cycles with one common vertex (path) then we use Lemma 10 and find a spanning subgraph with single maximal tree. Then after coloring edges of spanning subgraph by a color $\alpha$, we find a stable pair (beginnings of two incoming edges to the root of the tree).

The function TwoCyclesWithIntersection as a rule returns a pair of cycles with common vertex (path). The vast majority of digraphs contains such a pair of cycles. The goal of the subroutine is to omit the cubic complexity of the algorithm. The search of a stable pair is linear in this case and thus the whole algorithm is quadratic.

TwoCyclesWithIntersection (graph $G$ )

1 levels of all vertices first are negative

2 level $(\mathbf{r})=1$ and add $\mathbf{r}$ to stack

3 for every vertex $\mathbf{q}$ from stack

4 do

5 for every letter $\beta$

6 do

$7 \quad$ add $\mathbf{q} \beta$ to stack

$8 \quad$ if $\operatorname{level}(\mathbf{q} \beta) \geq 0$

$9 \quad \operatorname{level}(\mathbf{q} \beta)=\operatorname{level}(\mathbf{q})+1$

10 keep the cycle $\mathrm{C}$ of vertices $\mathbf{q} \beta, \mathbf{q}$ and break from both cycles

11 remove $\mathbf{q}$ from stack

12 for every vertex $\mathbf{r}$

13 do

14 if $\mathbf{r} \notin C$ level $((\mathbf{r})=-1$ (for a search of second cycle) 


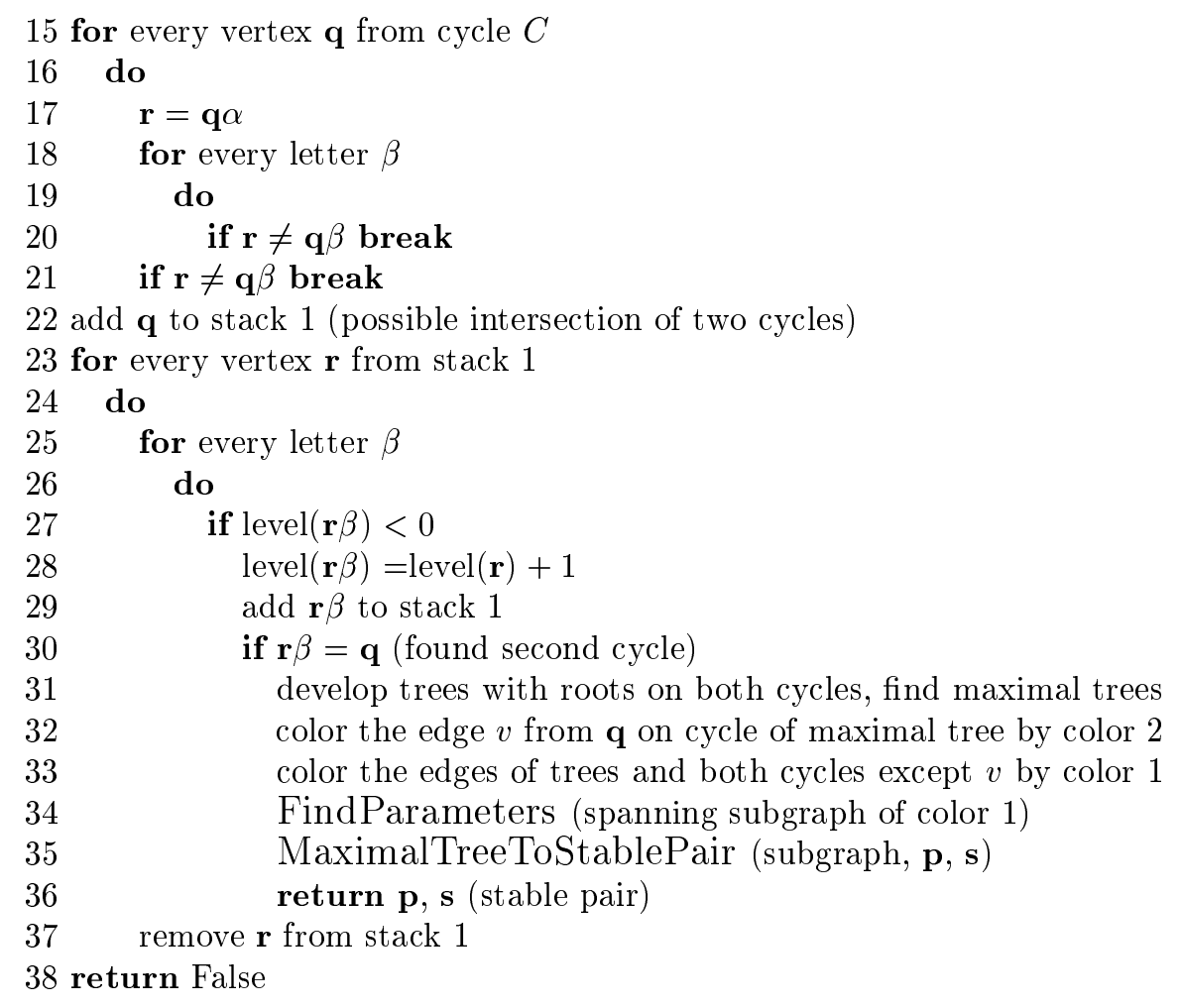

\subsection{The recoloring of the edges}

A repainting of the edges of the transition graph for to obtain a spanning subgraph with single maximal tree is a most complicated part of the algorithm. Let us fix the spanning subgraph $R$ of edges of a given color $\alpha$. We consider the flips from Lemmas 7 and 8 . The flips change $R$. According to the Lemmas, after at most $3 d$ steps either the number of edges in the cycles is growing or there exists a single maximal tree.

The subroutine of pseudo code Flips (spanning subgraph $F$ ) returns either a stable pair or enlarges the number of edges in cycles of the spanning subgraph. The subroutine uses linear subroutines FindParameters, MaximalTreeToStablePair and also has linear time complexity $O(n d)$.

We repeat the procedure with pseudo code Flips for a new graph if the number of edges in cycles after the flips grows. In the opposite case, we find a stable pair and then a homomorphic image of a smaller size. For a graph of given size, the complexity of this step is quadratic.

\subsection{Main procedure and complexity}

The Procedure Main uses all above-mentioned linear procedures and returns a synchronizing coloring (if exists) of the graph. 


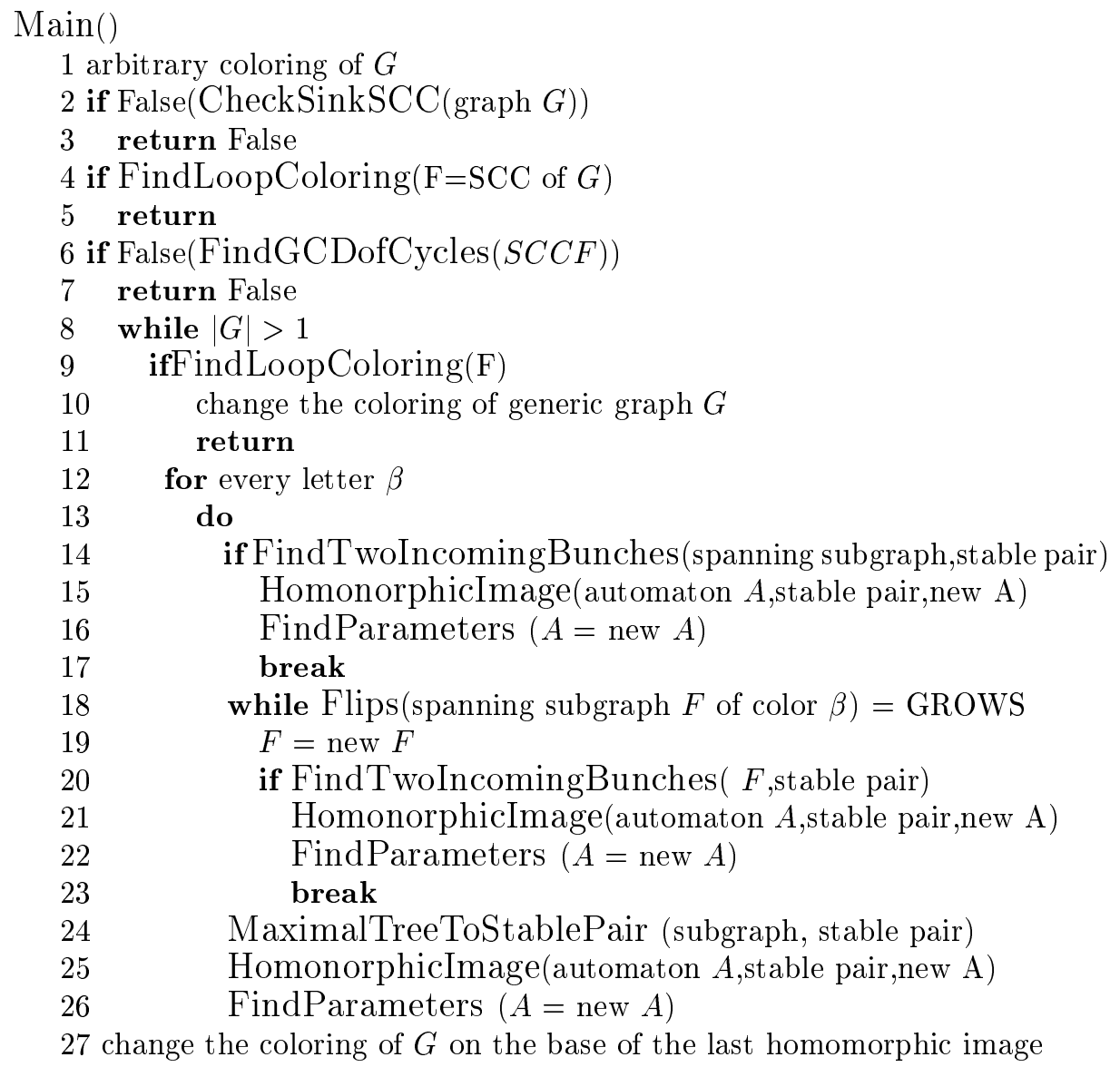

Some of above-mentioned linear subroutines are included in cycles on $n$ and $d$, sometimes twice on $n$. So the upper bound of the time complexity is $O\left(n^{3} d\right)$.

Nevertheless, the overall complexity of the algorithm in a majority of cases is $O\left(n^{2} d\right)$. The upper bound $O\left(n^{3} d\right)$ of the time complexity is reached only if the number of edges in the cycles grows slowly, the size of the automaton decreases also slowly, loops do not appear and the case of two ingoing bunches emerges rarely (the worst case). The space complexity is quadratic.

\section{References}

1. Adler, R.L.,Goodwyn, L.W., Weiss, B.: Equivalence of topological Markov shifts, Israel J. of Math., 27, 49-63 (1977).

2. Adler, R.L., Weiss, B.: Similarity of automorphisms of the torus, Memoirs of the Amer. Math. Soc., 98, Providence, RI, (1970).

3. Aho, A., Hopcroft, J., Ulman,J.: The Design and Analisys of Computer Algorithms, Addison-Wesley, (1974). 
4. Béal, M.P., Perrin, D.: A quadratic algorithm for road coloring. arXiv:0803.0726v2 [cs.DM] (2008).

5. Budzban, G., Mukherjea, A.: A semigroup approach to the Road Coloring Problem. Probability on Alg. Structures. Contemporary Mathematics, 261, 195-207, (2000).

6. Carbone, A.: Cycles of relatively prime length and the road coloring problem. Israel J. of Math., 123, 303-316, (2001).

7. Černy, J.: Poznamka $\mathrm{k}$ homogenym eksperimentom s konechnymi automatami, Math.-Fyz. Čas., 14, 208-215 (1964).

8. Černy, J., Piricka, A., Rosenauerova, B.: On directable automata, Kybernetika 7, 289-298 (1971).

9. Culik, K., Karhumaki, J., Kari, J.: A note on synchronized automata and Road Coloring Problem. Developments in Language Theory (5th Int. Conf., Vienna, 2001), J. of Found. Comput. Sci., 13, 459-471 (2002).

10. Friedman, J.: On the road coloring problem. Proc. of the Amer. Math. Soc., 110, 1133-1135 (1990).

11. Gocka, E., Kirchherr, W., Schmeichel, E.: A note on the road-coloring conjecture. Ars Combin., 49, 265-270, (1998).

12. Hegde, R., Jain, K.: Min-Max theorem about the Road Coloring Conjecture. EuroComb 2005, DMTCS proc., AE, 279 - 284, (2005).

13. Jonoska, N., Karl, S. A.: A molecular computation of the road coloring problem. DNA Based Computers II, DIMACS Series in DMTCS, 44, 87-96, (1998).

14. Kari, J.: Synchronizing finite automata on Eulerian digraphs. Lect. Notes in Comp. Sci., Springer, 2136, 432-438,( 2001).

15. Mateescu, A., Salomaa, A.: Many-Valued Truth Functions, Černy's Conjecture and Road Coloring. Bull. of European Ass. for TCS, 68, 134-148, (1999).

16. O'Brien, G.L.: The road coloring problem. Israel J. of Math., 39, 145-154, (1981).

17. Perrin, D., Schŭtzenberger, M.P.: Synchronizing prefix codes and automata, and the road coloring problem. In Symbolic Dynamics and Appl., Contemp. Math., 135, 295-318, (1992).

18. Rauff, J. V.: Way back from anywhere: exploring the road coloring conjecture. Math. and Comput. Education. 01,(2009).

19. Roman, A.: Decision Version of the Road Coloring Problem is NP-complete, Lect. Notes in Comp. Sci., 5699 287-297 (2009).

20. Rystsov, I. C.: Quasioptimal bound for the length of reset words for regular automata, Acta Cybernetica, 12, 145-152 (1995.)

21. Tarjan, R.E.: Depth first search and linear graph algorithms. SIAM J. Comput., 1, 146-160 (1972).

22. Trahtman, A.N.: A subquadratic algorithm for road coloring. arXiv:0801.2838v1 [cs.DM] (2008).

23. Trahtman, A.N.: Synchronizing Road Coloring., 5-th IFIP WCC-TCS, Springer, 273, 43-53 (2008).

24. Trahtman, A.N.: The road coloring problem. Israel Journal of Math., 172(1), 51-60 (2009).

25. A.N. Trahtman, Bauer T., Cohen N. Linear visualization of a Road Coloring. 9th Twente workshop on graphs and Comb. Optim., Cologne, 13-16 (2010). 